\title{
Call for Papers: „Schleier. Text-Bild-Ritual“
}

Vom 29. 11.-2. 12. 2001 findet an der Universität Trier ein internationales und interdisziplinäres Symposion zum Thema: „Schleier. Text-Bild-Ritual“ statt. Es sollen unterschiedliche Konzeptionen des "Schleiers“ in den text-, bild- und kulturgeschichtlichen Medien der verschiedenen Zeiten, aber auch innerhalb neuerer und neuester Wissenschaftsdiskurse untersucht werden. Der Vielfalt dieser Konzeptionen gemäß, ist ein breites methodisches Spektrum erwünscht. Deshalb sind sowohl Vertreter und Vertreterinnen der Literaturwissenschaft, der Kunstgeschichte und der Theaterwissenschaft als auch der Philosophie, der Theologien und der Ethnologie sowie anderer Fächer zur Beteiligung aufgerufen.

Vielversprechende Schleier-Kontexte sollten u.a. in Magie und Zauber, in Mode und Körperinszenierung, in Erotik und Fetischismus wie auch in den Nachbardiskursen des Heiligen, der Wahrheit, der Nacktheit und der Natur auszumachen sein. Immer aber dürften Fragen und Probleme sowohl der Identitäts- als auch der Geschlechterkonstitution betroffen sein.

Themenvorschläge und Abstracts (1 Seite) zu diesem weiten Problemkreis werden binnen vier Wochen erbeten an:

Prof. Dr. Gerhard Wolf

Kunstgeschichte

Universität Trier

54286 Trier

Email: wolf@uni-trier.de

oder an:

Dr. Johannes Endres

Institut für Germanistik

Universität Leipzig

(Nur!) Email: endres@rz.uni-leipzig.de 